Pacific

Journal of

Mathematics

\title{
A PLANAR SOBOLEV EXTENSION THEOREM FOR PIECEWISE LINEAR HOMEOMORPHISMS
}

EMANUELA RADICI 


\title{
A PLANAR SOBOLEV EXTENSION THEOREM FOR PIECEWISE LINEAR HOMEOMORPHISMS
}

\author{
EMANUELA RADICI
}

\begin{abstract}
We prove that a planar piecewise affine homeomorphism $\varphi$ defined on the boundary of the square can be extended to a piecewise affine homeomorphism $h$ of the whole square, in such a way that $\|h\|_{W^{1, p}}$ is bounded from above by $\|\varphi\|_{W^{1, p}}$ for every $p \geq 1$.
\end{abstract}

\section{Introduction}

Let $\mathcal{Q}$ be the unit square in $\mathbb{R}^{2}$ and $\varphi$ be a piecewise affine homeomorphism with finitely many affine components that maps $\partial \mathcal{Q}$ to a closed curve in $\mathbb{R}^{2}$. We call a piecewise affine map with finitely many affine components a finitely piecewise affine map. In this work, we provide a general recipe for extending $\varphi$ to a function $h$ of the whole square which maintains the finitely piecewise affine structure and whose Sobolev $W^{1, p}$-norm is controlled from above by $\|\varphi\|_{W^{1, p}}$. That such an extension exists is well known, and its construction is not difficult, but showing the existence of an extension with good control on its norm is a substantial problem. In fact, we will establish a bound

$$
\|D h\|_{L^{p}(\mathcal{Q})} \leq K\|D \varphi\|_{L^{p}(\partial \mathcal{Q})}
$$

for a suitable geometric constant $K$ which depends only on $p$. It is appropriate to explain briefly the context of our work and its utility. The problem of finding approximations of a planar homeomorphism $f: \Omega \rightarrow \mathbb{R}^{2}$ has a long history in the literature and recently it was realized to be relevant to the study of the regularity of minimizers for standard energies in the area of nonlinear elasticity. Many important results are already available on this topic. See, for instance, [Mora-Corral 2009; Bellido and Mora-Corral 2011; Iwaniec et al. 2011; Daneri and Pratelli 2015; Hencl and Pratelli 2015] for an overview of what is known. Let us recall the approach introduced in the last two of these references, where the authors create the approximation step by step, starting from an explicit subdivision of the domain $\Omega$ that depends on the Lebesgue points of $D f$. Although their settings and the regularity of their approximations are very different, in both papers the strategy

MSC2010: 46E35.

Keywords: piecewise affine approximation, Sobolev homeomorphisms. 
is to split the domain into a countable disjoint union of simple polygons (in general triangles or squares) by introducing a locally finite one-dimensional grid, which consists of the union of the boundaries of such polygons. A first piecewise linear approximation of $f$ is defined on the one-dimensional grid and, only in a second step, the approximation is extended in the interior of each simple polygon, being careful to get the regularity claimed. In this work we focus on the second step of this strategy, namely we already assume the existence of a piecewise linear function $f_{\varepsilon}$ defined on a locally finite grid of squares, let us call it $\Delta$, and we give all the ingredients needed for extending $f_{\varepsilon}$ to a piecewise affine function of the whole domain $\Omega$ with suitably small $W^{1, p}$-norm. In fact, the extension of $f_{\varepsilon}$ into a single square $\mathcal{Q}$ involves only the values on the boundary $\partial \mathcal{Q}$ of $\mathcal{Q}$, and it is useful to have an estimate like

$$
\left\|f_{\varepsilon}\right\|_{W^{1, p}(\mathcal{Q})}<K\left\|f_{\varepsilon}\right\|_{W^{1, p}(\partial \mathcal{Q})},
$$

for a suitable constant $K$. Let us already say that our proof does not depend on the precise value of $p$, thus it holds true for every $p \in[1, \infty)$. An analogous result was already proved in the cases $p=\infty$ in [Daneri and Pratelli 2015] and $p=1$ in [Hencl and Pratelli 2015], while in this work we generalize to any $p>1$ a technique introduced in [Hencl and Pratelli 2015]. Furthermore, an extension of this result seems to be true also in the Orlicz-Sobolev spaces (see [Campbell 2015]). For us, $\mathcal{Q}$ will be the rotated square centered in the origin and with corners in $( \pm 1,0),(0, \pm 1)$. Our result is the following.

Theorem 1.1. Let $\varphi: \partial \mathcal{Q} \rightarrow \mathbb{R}^{2}$ be a one-to-one piecewise affine function. Then there exists a finitely piecewise affine homeomorphism $h: \mathcal{Q} \rightarrow \mathbb{R}^{2}$ that satisfies $h \equiv \varphi$ on $\partial \mathcal{Q}$ and, for any $p \geq 1$, there is a constant $K$ depending only on $p$ such that the estimate

$$
\int_{\mathcal{Q}}|D h(x)|^{p} d x \leq K \int_{\partial \mathcal{Q}}|D \varphi(t)|^{p} d \mathcal{H}^{1}(t)
$$

holds.

The plan of the paper is very simple: in the following section we make a short remark about the notation, the second section is devoted to the proof of Theorem 1.1 and in the last remark we explain the case of a generic square in the plane.

Notation. Let us briefly introduce the notation we use throughout the paper. We call $\mathcal{Q}=\left\{(x, y) \in \mathbb{R}^{2}:|x|+|y|<1\right\}$ the rotated square centered in the origin and $\mathcal{Q}$ the image through $h$ of $\mathcal{Q}$. With the capital letters $A, B$ we always refer to points lying on the boundary of $\mathcal{Q}$, while $P$ and $R$ denote points in the interior of $\mathcal{Q}$. The points in $\partial \mathcal{Q}$ and in the interior of $\mathcal{Q}$ will be denoted similarly in bold style: $\boldsymbol{A}, \boldsymbol{B}, \boldsymbol{P}, \boldsymbol{R}$. When we use the same letter in normal and bold style, for example $A$ and $\boldsymbol{A}$, this always means that $\boldsymbol{A}$ is the image of $A$ through the mapping 
that we are considering in that moment. By $A B$ and $A B C$ we mean, respectively, the segment between $A$ and $B$ and the triangle of corners $A, B$ and $C$ (the same also for $\boldsymbol{A B}, \boldsymbol{A B C}$ ). The modulus of the horizontal and vertical derivatives of a function $f=\left(f_{1}, f_{2}\right): \mathbb{R}^{2} \rightarrow \mathbb{R}^{2}$ is denoted as

$$
\left|D_{1} f\right|=\sqrt{\left(\frac{\partial f_{1}}{\partial x}\right)^{2}+\left(\frac{\partial f_{2}}{\partial x}\right)^{2}}, \quad\left|D_{2} f\right|=\sqrt{\left(\frac{\partial f_{1}}{\partial y}\right)^{2}+\left(\frac{\partial f_{2}}{\partial y}\right)^{2}},
$$

and with the symbol $\mathcal{H}^{1}$ we indicate the standard 1-dimensional Hausdorff measure. Finally, the letter $K$ will always indicate a purely geometric constant that depends only on $p$. Since it is the existence, not the size, of $K$ that matters, we do not calculate the explicit value of $K$ but we show at each step that it remains finite and it stays independent from all the parameters but $p$.

\section{Proof of Theorem 1.1}

We generalize to every $1 \leq p<\infty$ a strategy introduced in [Hencl and Pratelli 2015] for $p=1$. To keep this work self contained, we present also the parts of the proof stated in [Hencl and Pratelli 2015] that do not depend on the exponent $p$.

Proof of Theorem 1.1. Since the proof of Theorem 1.1 is long, we split it into several steps for sake of clarity.

Step I. Choice of corners. It is useful to know that $|D \varphi|^{p}$ does not critically accumulate around the two opposite corners of $\partial \mathcal{Q}$, which we denote as $V_{1} \equiv(0,-1)$ and $V_{2} \equiv(0,1)$. More precisely, the estimate we would like to have is the following:

(2-1) $\int_{B\left(V_{i}, r\right) \cap \partial \mathcal{Q}}|D \varphi|^{p} d \mathcal{H}^{1} \leq \widetilde{K} r \int_{\partial \mathcal{Q}}|D \varphi|^{p} d \mathcal{H}^{1} \quad$ for all $r \in(0, \sqrt{2}), i \in\{1,2\}$.

This is not true for a generic $\varphi$, but in this step we show that there always exists a pair of opposite points $P_{1}, P_{2}$ on $\partial \mathcal{Q}$ that satisfies (2-1) in place of $V_{1}, V_{2}$ with $\widetilde{K}=6$. Notice that it is always possible to apply a bi-Lipschitz transformation of $\partial \mathcal{Q}$ to itself which moves such $P_{1}, P_{2}$ to $V_{1}, V_{2}$ respectively. Since the bi-Lipschitz constant does not depend on the particular values of $P_{1}$ and $P_{2}$, then estimate (2-1) follows straightforwardly for a suitable constant $\widetilde{K}$. Thanks to this observation, the step is concluded once we prove the existence of such $P_{1}, P_{2}$. Two generic opposite points are a good choice unless at least one of them is in the set

$$
\mathcal{A}=\left\{A \in \partial \mathcal{Q}: \int_{B(A, r) \cap \partial \mathcal{Q}}|D \varphi|^{p} d \mathcal{H}^{1}>6 r \int_{\partial \mathcal{Q}}|D \varphi|^{p} d \mathcal{H}^{1} \text { for some } r \in(0, \sqrt{2})\right\} .
$$

Thus, the existence of a good pair $P_{1}, P_{2}$ is established if $\mathcal{H}^{1}(\mathcal{A})$ is not too big. By a Vitali covering argument, it is always possible to cover $\mathcal{A}$ with a countable 


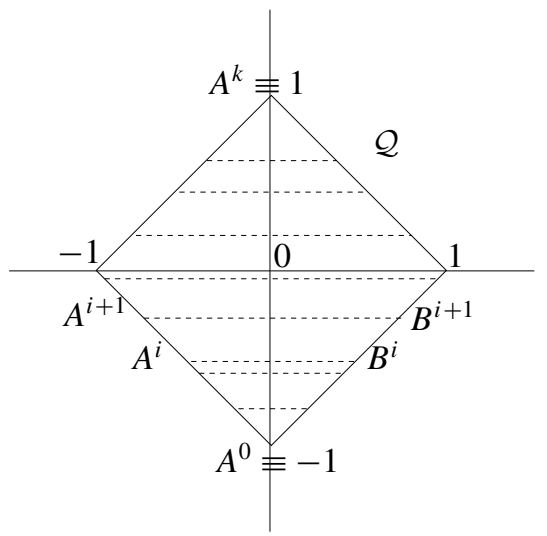

(a)

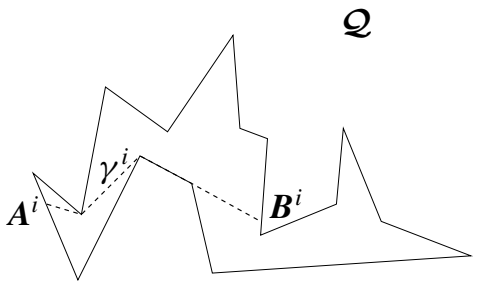

(b)

Figure 1. (a) A grid on $\mathcal{Q}$ and (b) a path $\gamma^{i}$ in $\mathcal{Q}$.

union of balls $B\left(A_{i}, 3 r_{i}\right)$ such that $A_{i} \in \mathcal{A}$ and the sets $B\left(A_{i}, r_{i}\right) \cap \partial Q$ are pairwise disjoint. In particular, one has

$$
\mathcal{H}^{1}(\mathcal{A}) \leq \sum_{i} 6 r_{i} \leq \sum_{i} \frac{\int_{B\left(A, r_{i}\right) \cap \partial \mathcal{Q}}|D \varphi|^{p} d \mathcal{H}^{1}}{\int_{\partial \mathcal{Q}}|D \varphi|^{p} d \mathcal{H}^{1}} \leq 1,
$$

thus implying the existence of a pair of opposite points, $P_{1}$ and $P_{2}$ in $\partial \mathcal{Q} \backslash \mathcal{A}$, satisfying (2-1).

Step II. Construction of one-dimensional grids in $\mathcal{Q}$ and in $\mathcal{Q}$. Let us denote with $\mathcal{Q}$ the bounded component of $\mathbb{R}^{2} \backslash \varphi(\partial \mathcal{Q})$, which is a polygon because $\varphi$ is piecewise affine. Notice that the problem of finding a piecewise affine homeomorphism $h$ which maps $\mathcal{Q}$ into $\mathcal{Q}$ makes sense. Since we want $h \equiv \varphi$ on $\partial \mathcal{Q}$, our approach is the following: we start extending the function $\varphi$ on a suitable one-dimensional grid on $\mathcal{Q}$, we then "complete" this grid to obtain a triangulation of $\mathcal{Q}$ and, at the end, we define $h$ inside each triangle of the triangulation as the affine function extending the values on the boundary. In this step, we introduce the one-dimensional grid in $\mathcal{Q}$ and we construct a related grid in $\mathcal{Q}$, which will be the image through $h$ of the grid on $\mathcal{Q}$. For the construction, we make use of several horizontal segments $A^{i} B^{i}$ whose endpoints are in $\partial \mathcal{Q}$. We call $A^{i}=\left(A_{1}^{i}, A_{2}^{i}\right)$ the endpoint that has negative first component and we choose the indexes $i$ so that $A_{2}^{i}$ increases with respect to $i$ from -1 to 1 (see Figure 1(a)). It is convenient to include also $V_{1}, V_{2}$ in the grid, therefore we denote them consistently as $A^{0} \equiv B^{0} \equiv V_{1} \equiv(0,-1)$ and $A^{k} \equiv B^{k} \equiv V_{2} \equiv(0,1)$. We consider many horizontal segments $A^{i} B^{i}$ such that for every $i$ the restriction of $\varphi$ to $A^{i} A^{i+1}$ or $B^{i} B^{i+1}$ is linear. Notice that this property is still valid even if we take more horizontal segments $A^{i} B^{i}$, therefore, we are allowed to add points $A^{i}$ and $B^{i}$ during the construction (of course, being careful 
in adding only finitely many). Once the grid on $\mathcal{Q}$ is fixed, we define a second one, this time on $\mathcal{Q}$, that is the union of the geodesics $\gamma^{i}$ inside $\overline{\mathcal{Q}}$ connecting $\varphi\left(A^{i}\right)$ and $\varphi\left(B^{i}\right)$. Since $\mathcal{Q}$ is a polygon, $\gamma^{i}$ is piecewise affine and, moreover, the junction between any two consecutive affine pieces of $\gamma^{i}$ lies in $\partial \mathcal{Q}$ (see Figure 1(b)). In order to simplify the notation, we write the points of $\mathcal{Q}$ in bold style: $\boldsymbol{A}^{i} \equiv \varphi\left(A^{i}\right)$ and $\boldsymbol{B}^{i} \equiv \varphi\left(B^{i}\right)$. Up to adding points between $A^{0}$ and $A^{1}$ (we do the same also for $A^{k-1}$ and $A^{k}$ ), we can always assume that $\gamma^{1}$ (resp. $\gamma^{k-1}$ ) is either a segment $\boldsymbol{A}^{1} \boldsymbol{B}^{1}$ (resp. $\boldsymbol{A}^{k-1} \boldsymbol{B}^{k-1}$ ), or it is the union of the two segments $\boldsymbol{A}^{1} \boldsymbol{A}^{0}$ and $\boldsymbol{A}^{0} \boldsymbol{B}^{1}$ (resp. $\boldsymbol{A}^{k-1} \boldsymbol{A}^{k}$ and $\boldsymbol{A}^{k} \boldsymbol{B}^{k-1}$ ) lying entirely in $\partial \mathcal{Q}$. It is well known in the literature that the shortest path that connects two generic points inside a simply connected set is unique. In particular, this result ensures that the paths $\gamma^{i}$ are unique and implies that the grid on $\mathcal{Q}$ is well defined.

Step III. Relevant properties of the paths $\gamma^{i}$. In this step we present some properties of the paths $\gamma^{i}$. The first property is a consequence of the uniqueness: whenever two paths $\gamma^{i+1}$ and $\gamma^{i}$ intersect each other they coincide from the first to the last point of intersection. In particular, $\gamma^{i+1}$ and $\gamma^{i}$ cannot cross each other, thus allowing us to distinguish three different parts on each path

$$
\gamma^{i}=\gamma_{1}^{i} \cup \gamma_{2}^{i} \cup \gamma_{3}^{i}, \quad \gamma^{i+1}=\gamma_{1}^{i+1} \cup \gamma_{2}^{i+1} \cup \gamma_{3}^{i+1}
$$

In detail, if $\boldsymbol{A}$ and $\boldsymbol{B}$ are the first and last points, respectively, of the common part between $\gamma^{i}$ and $\gamma^{i+1}$, we call $\gamma_{1}^{i}$ the path from $\boldsymbol{A}^{i}$ to $\boldsymbol{A}$ (analogous for $\gamma_{1}^{i+1}$ ), $\gamma_{2}^{i}$ the path from $\boldsymbol{A}$ to $\boldsymbol{B}\left(\gamma_{2}^{i} \equiv \gamma_{2}^{i+1}\right)$ and $\gamma_{3}^{i}$ the last part of the path from $\boldsymbol{B}$ to $\boldsymbol{B}^{i}$ (analogous for $\gamma_{3}^{i+1}$ ). When $\gamma^{i}$ and $\gamma^{i+1}$ have no intersection, we directly set $\gamma^{i} \equiv \gamma_{1}^{i}$ and $\gamma^{i+1} \equiv \gamma_{1}^{i+1}$, while $\gamma_{2}^{i}, \gamma_{3}^{i}, \gamma_{2}^{i+1}, \gamma_{3}^{i+1}$ are empty paths. Let us observe that such subdivision of $\gamma^{i}$ is related to the curve $\gamma^{i+1}$ and there is no reason why it should match with the one related to $\gamma^{i-1}$. The last property of paths $\gamma^{i}$ that we recall is fundamental for showing estimate (1-1) (for a formal proof see Step 5 of Theorem 2.1 in [Hencl and Pratelli 2015]). Let $\boldsymbol{P}$ be the last point of the curve $\gamma_{1}^{i+1}$; no matter whether $\boldsymbol{P}$ coincides with $\boldsymbol{A}$ or $\boldsymbol{B}^{i+1}$, the polygon having boundary $\gamma_{1}^{i+1} \cup \boldsymbol{A}^{i+1} \boldsymbol{P}$ is always convex (see Figure 2).

Step IV. Triangular grid on $\mathcal{Q}$ and estimate on "vertical sides". In this step we are going to select finitely many points on the paths $\gamma^{i}$ in order to get a triangular grid on $\mathcal{Q}$. For all $i=1, \ldots, k-2$, we call $\mathcal{D}_{i}$ the closure of the polygon having boundary $\boldsymbol{A}^{i} \boldsymbol{A}^{i+1} \cup \gamma^{i} \cup \gamma^{i+1} \cup \boldsymbol{B}^{i} \boldsymbol{B}^{i+1}$ (which lies inside the closure of $\mathcal{Q}$ ), then we argue separately for each single $\mathcal{D}_{i}$. For every "strip" $\mathcal{D}_{i}$ we select some points on $\gamma^{i}, \gamma^{i+1}$ depending on the relation between $\gamma_{1}^{i}, \gamma_{2}^{i}, \gamma_{3}^{i}$ and $\gamma_{1}^{i+1}, \gamma_{2}^{i+1}, \gamma_{3}^{i+1}$. We argue separately for the cases $\gamma^{i} \cap \gamma^{i+1} \neq \varnothing$ and $\gamma^{i} \cap \gamma^{i+1}=\varnothing$. Both situations are depicted in Figure 3. 


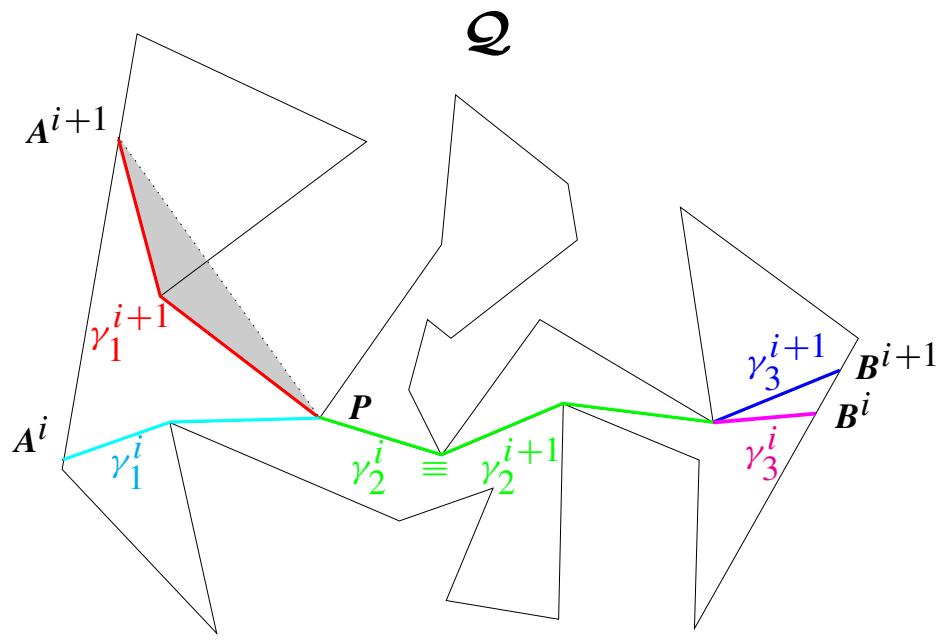

Figure 2. Convexity of the polygon delimited by $\gamma_{1}^{i+1} \cup \boldsymbol{A}^{i+1} \boldsymbol{P}$.

Let us start from the nonempty case (Figure 3(a)). For any endpoint of a linear piece of $\gamma_{1}^{i+1}$ or $\gamma_{1}^{i}$ we consider the corresponding point on the other path so that the segment connecting the two points is parallel to $\boldsymbol{A}^{i} \boldsymbol{A}^{i+1}$. We denote with $\boldsymbol{P}_{j}$ the points taken on $\gamma_{1}^{i+1}$ and with $\boldsymbol{R}_{j}$ the corresponding point on $\gamma_{1}^{i}$. Notice that the convexity result introduced in Step III ensures that the segment $\boldsymbol{P}_{j} \boldsymbol{R}_{j}$ is always well defined and, moreover, it satisfies $\mathcal{H}^{1}\left(\boldsymbol{P}_{j} \boldsymbol{R}_{j}\right) \leq \mathcal{H}^{1}\left(\boldsymbol{A}^{i} \boldsymbol{A}^{i+1}\right)$, thus, in particular,

$$
\mathcal{H}^{1}\left(\boldsymbol{P}_{j} \boldsymbol{R}_{j}\right) \leq \max \left\{\mathcal{H}^{1}\left(\boldsymbol{A}^{i} \boldsymbol{A}^{i+1}\right), \mathcal{H}^{1}\left(\boldsymbol{B}^{i} \boldsymbol{B}^{i+1}\right)\right\} .
$$

With a symmetric strategy we select other points $\boldsymbol{P}_{j}$ and $\boldsymbol{R}_{j}$ on $\gamma_{3}^{i+1}$ and $\gamma_{3}^{i}$, respectively, by taking this time $\boldsymbol{P}_{j} \boldsymbol{R}_{j}$ parallel to $\boldsymbol{B}^{i} \boldsymbol{B}^{i+1}$. Let us recall that (2-2) still holds true in this case, since now $\mathcal{H}^{1}\left(\boldsymbol{P}_{j} \boldsymbol{R}_{j}\right) \leq \mathcal{H}^{1}\left(\boldsymbol{B}^{i} \boldsymbol{B}^{i+1}\right)$. Finally, in the

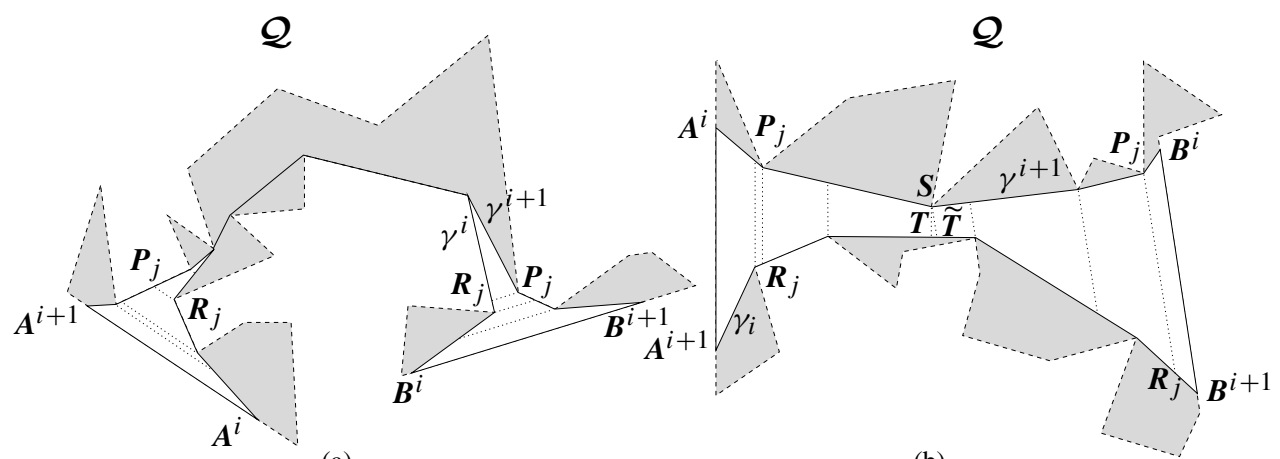

(a)

(b)

Figure 3. Selection of points on $\gamma^{i}$ and $\gamma^{i+1}$. 
common part $\gamma_{2}^{i} \equiv \gamma_{2}^{i+1}$, we take all the endpoints of the linear pieces of the path and by construction $\boldsymbol{P}_{j} \equiv \boldsymbol{R}_{j}$. Of course, (2-2) is trivially true because in this case $\mathcal{H}^{1}\left(\boldsymbol{P}_{j} \boldsymbol{R}_{j}\right)=0$. If $\gamma^{i} \cap \gamma^{i+1}=\varnothing$, the strategy is a little bit different (see Figure 3(b)). In the specific case in which $\boldsymbol{A}^{i} \boldsymbol{A}^{i+1}$ and $\boldsymbol{B}^{i} \boldsymbol{B}^{i+1}$ are parallel to each other, we can argue exactly as in the previous case: therefore all the points $\boldsymbol{P}_{j}$ selected on $\gamma^{i+1}$ can be either endpoints of linear pieces of $\gamma^{i+1}$ or the corresponding point of $\boldsymbol{R}_{j}$, where $\boldsymbol{R}_{j}$ is an endpoint for $\gamma^{i}$. Moreover, by construction, $\boldsymbol{P}_{j} \boldsymbol{R}_{j}$ is always parallel to both $\boldsymbol{A}^{i} \boldsymbol{A}^{i+1}$ and $\boldsymbol{B}^{i} \boldsymbol{B}^{i+1}$ and (2-2) is still satisfied thanks to Step III. For generic $\boldsymbol{A}^{i} \boldsymbol{A}^{i+1}$ and $\boldsymbol{B}^{i} \boldsymbol{B}^{i+1}$ we argue as follows. By symmetry, it is not restrictive to assume that $\boldsymbol{A}^{i} \boldsymbol{A}^{i+1}$ is vertical and the two lines with directions $\boldsymbol{A}^{i} \boldsymbol{A}^{i+1}$ and $\boldsymbol{B}^{i} \boldsymbol{B}^{i+1}$ intersect in a point that is closer to $\boldsymbol{A}^{i+1}$ and $\boldsymbol{B}^{i+1}$ than $\boldsymbol{A}^{i}$ and $\boldsymbol{B}^{i}$ (as shown in Figure 3(b)). Let $\boldsymbol{S}, \boldsymbol{T}, \tilde{\boldsymbol{T}}$ be three points such that $\boldsymbol{S}$ is on $\gamma^{i+1}$ and $\boldsymbol{T}, \tilde{\boldsymbol{T}}$ are on $\gamma^{i}$ and $\boldsymbol{S T}$ is the shortest segment inside $\mathcal{D}_{i}$ which is parallel to $\boldsymbol{A}^{i} \boldsymbol{A}^{i+1}$ (notice that $\boldsymbol{S}$ can even happen to be $\boldsymbol{A}^{i+1}$ or $\boldsymbol{B}^{i+1}$ ) while $\tilde{\boldsymbol{T}}$ is the intersection between $\gamma^{i}$ and the half line starting from $\boldsymbol{S}$ with direction $\boldsymbol{B}^{i} \boldsymbol{B}^{i+1}$. On one side, with the usual strategy, we select points $\boldsymbol{P}_{j}, \boldsymbol{R}_{j}$, with $\boldsymbol{P}_{j}$ between $\boldsymbol{A}^{i+1}$ and $\boldsymbol{S}$ and $\boldsymbol{R}_{j}$ between $\boldsymbol{A}^{i}$ and $\boldsymbol{T}$, so that $\boldsymbol{P}_{j} \boldsymbol{R}_{j}$ is parallel to $\boldsymbol{A}^{i} \boldsymbol{A}^{i+1}$. On the other side, we take $\boldsymbol{P}_{j}, \boldsymbol{R}_{j}$ with $\boldsymbol{P}_{j}$ between $\boldsymbol{S}$ and $\boldsymbol{B}^{i+1}, \boldsymbol{R}_{j}$ between $\tilde{\boldsymbol{T}}$ and $\boldsymbol{B}^{i}$, and $\boldsymbol{P}_{j} \boldsymbol{R}_{j}$ parallel to $\boldsymbol{B}^{i} \boldsymbol{B}^{i+1}$. Finally, to any endpoint $\boldsymbol{R}_{j}$ of a linear piece of $\gamma^{i}$ that happens to be between $\boldsymbol{T}$ and $\tilde{\boldsymbol{T}}$, we associate $\boldsymbol{P}_{j} \equiv \boldsymbol{S}$. Notice that, by construction, estimate (2-2) is satisfied. We can then introduce the triangular grid on $\mathcal{D}_{i}$ as the union of the boundaries of the triangles $\boldsymbol{P}_{j} \boldsymbol{P}_{j+1} \boldsymbol{R}_{j}$ and $\boldsymbol{P}_{j+1} \boldsymbol{R}_{j} \boldsymbol{R}_{j+1}$ that are not degenerate. Moreover, recalling Step II, the polygons delimited by $\gamma^{1} \cup \boldsymbol{A}^{1} \boldsymbol{A}^{0} \cup \boldsymbol{A}^{0} \boldsymbol{B}^{1}$ and $\gamma^{k-1} \cup \boldsymbol{A}^{k-1} \boldsymbol{A}^{k} \cup \boldsymbol{A}^{k} \boldsymbol{B}^{k-1}$ are either triangles themselves or the union of two segments lying in $\partial \mathcal{Q}$, thus we actually defined a triangular grid on the whole $\mathcal{Q}$ and this concludes Step IV.

Step $V$. Triangular grid on $\mathcal{Q}$ and definition of $\tilde{h}$. We recall that our aim is to define

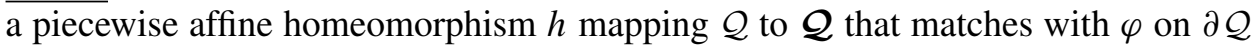
and satisfies estimate (1-1). In this step, we construct a function $\tilde{h}: \mathcal{Q} \rightarrow \mathcal{Q}$ which is piecewise affine and coincides with $\varphi$ on the boundary of $\mathcal{Q}$ and in Steps VI, VII we will prove that estimate (1-1) is satisfied by $\tilde{h}$. Let us already remark that in general $\tilde{h}$ will not be a one-to-one function, therefore we will have to suitably modify it later to obtain the homeomorphism $h$. We split the construction into three steps: first, we define the function $\tilde{h}$ only on $\partial \mathcal{Q}$ and on the segments $A^{i} B^{i}$, we then make use of the triangular grid of $\mathcal{Q}$ defined in Step IV to find a triangular grid on $\mathcal{Q}$ and, finally, we use these triangular grids to extend $\tilde{h}$ on the whole $\mathcal{Q}$. We start by taking $\tilde{h} \equiv \varphi$ on $\partial \mathcal{Q}$ such that it maps all the horizontal segments $A^{i} B^{i}$ to the respective path $\gamma^{i}$ parametrized at constant speed. Notice that, in this way, $\tilde{h}$ is continuous and piecewise linear. Recalling the notation introduced in the previous step, we focus then on the polygon $\mathcal{D}_{i}$ : we associate to any $\boldsymbol{P}_{j}$ on $\gamma^{i+1}$ the point $P_{j}$ on $A^{i+1} B^{i+1}$ such that $\boldsymbol{P}_{j}=\tilde{h}\left(P_{j}\right)$, and to any $\boldsymbol{R}_{j}$ on $\gamma^{i}$ the 
point $R_{j}$ on $A^{i} B^{i}$ such that $\boldsymbol{R}_{j}=\tilde{h}\left(R_{j}\right)$. For the sake of clarity we denote the endpoints $A^{i} A^{i+1} B^{i+1} B^{i}$ consistently with the notation, namely we call $P_{0} \equiv A^{i+1}$, $P_{N} \equiv B^{i+1}, R_{0} \equiv A^{i}$ and $R_{N} \equiv B^{i}$ for a suitable $N$. We can now define $\tilde{h}$ on the strip $\mathcal{D}_{i}:=A^{i} A^{i+1} \cup A^{i+1} B^{i+1} \cup B^{i} B^{i+1} \cup A^{i} B^{i}$ as the function which is affine on each of the triangles $P_{j} P_{j+1} R_{j}$ or $P_{j+1} R_{j} R_{j+1}$ (notice that clearly $\tilde{h}$ can be degenerate on some triangles). In more detail, we define $\tilde{h}$ on $P_{j} P_{j+1} R_{j}$ as the affine function which maps $P_{j} P_{j+1} R_{j}$ onto $\boldsymbol{P}_{j} \boldsymbol{P}_{j+1} \boldsymbol{R}_{j}$ extending the values on the boundary (the very same definition is used for triangles of the form $P_{j+1} R_{j} R_{j+1}$ ). It still remains to define $\tilde{h}$ on the top and bottom triangles of $\mathcal{Q}$. Let us consider the bottom triangle $A^{0} A^{1} B^{1}$ (the definition is symmetric for $A^{k} A^{k-1} B^{k-1}$ ); then we know from Step II that $\gamma^{1}$ can be either a segment $\boldsymbol{A}^{1} \boldsymbol{B}^{1}$ or the union of two segments $\boldsymbol{A}^{0} \boldsymbol{A}^{1}, \boldsymbol{A}^{0} \boldsymbol{B}^{1}$ laying on $\partial \mathcal{Q}$. In the first case we again define $\tilde{h}$ as the affine function that extends the values on the boundary. In the other case we will subdivide $A^{0} A^{1} B^{1}$ into two triangles $A^{0} A^{1} P$ and $A^{0} P B^{1}$, where $P$ is the point on $A^{1} B^{1}$ such that $\tilde{h}(P)=\boldsymbol{A}^{0}$, then we define $\tilde{h}$ to be constantly equal to $\boldsymbol{A}^{0}$ on the segment $P A^{0}$ and the degenerate affine function extending the values on the boundary on $A^{0} A^{1} P$ and $A^{0} P B^{1}$. By construction, the function $\tilde{h}$ is piecewise affine, coincides with $\varphi$ on $\partial \mathcal{Q}$ and it is also continuous.

Step VI. Estimate for $\int_{A^{0} A^{1} B^{1}}|D \tilde{h}|^{p}$. As mentioned above, this step and the following one are devoted to showing that the function $\tilde{h}$ satisfies the estimate (1-1). Here, in particular, we focus on the bottom triangle $\mathcal{T}:=A^{0} A^{1} B^{1}$ (the very same argument holds also for the top triangle $A^{k} A^{k-1} B^{k-1}$ ), and we prove that

$$
\int_{\mathcal{T}}|D \tilde{h}|^{p} \leq K_{1} \int_{\partial Q}|D \varphi|^{p} d \mathcal{H}^{1}
$$

where $K_{1}$ denotes a purely geometric constant. Recall that, by definition, $\tilde{h}(\mathcal{T})$ is either the nondegenerate triangle $\boldsymbol{A}^{0} \boldsymbol{A}^{1} \boldsymbol{B}^{1}$ or the union of the two segments $\boldsymbol{A}^{1} \boldsymbol{A}^{0} \cup \boldsymbol{A}^{0} \boldsymbol{B}^{1}$. In the nondegenerate case, $D \tilde{h}$ is constant on $\mathcal{T}$, therefore we denote its constant value with $D^{b} \tilde{h}=\left(D_{1}^{b} \tilde{h}, D_{2}^{b} \tilde{h}\right)$. Moreover, by construction, $\tilde{h}\left(A^{0} A^{1}\right)=\boldsymbol{A}^{0} \boldsymbol{A}^{1}$ and $\tilde{h}\left(A^{0} B^{1}\right)=\boldsymbol{A}^{0} \boldsymbol{B}^{1}$, thus we can write the following relations:

$$
\frac{\sqrt{2}}{2}\left|D_{1}^{b} \tilde{h}+D_{2}^{b} \tilde{h}\right|=\frac{\mathcal{H}^{1}\left(\boldsymbol{A}^{0} \boldsymbol{B}^{1}\right)}{\mathcal{H}^{1}\left(A^{0} B^{1}\right)}, \quad \frac{\sqrt{2}}{2}\left|-D_{1}^{b} \tilde{h}+D_{2}^{b} \tilde{h}\right|=\frac{\mathcal{H}^{1}\left(\boldsymbol{A}^{0} \boldsymbol{A}^{1}\right)}{\mathcal{H}^{1}\left(A^{0} A^{1}\right)},
$$

which, in turn, imply that

$$
\left|D^{b} \tilde{h}\right|^{p} \leq \frac{2^{p} \mathcal{H}^{1}\left(\boldsymbol{A}^{0} \boldsymbol{A}^{1}\right)^{p}+2^{p} \mathcal{H}^{1}\left(\boldsymbol{A}^{0} \boldsymbol{B}^{1}\right)^{p}}{\mathcal{H}^{1}\left(A^{0} A^{1}\right)^{p}},
$$

where we used the fact that $\mathcal{H}^{1}\left(A^{0} A^{1}\right)=\mathcal{H}^{1}\left(A^{0} B^{1}\right)$. On the other hand, we have that $|D \varphi|$ is constant on each of the segments $A^{0} A^{1}, A^{0} B^{1}$ and this gives us an 
estimate on $\mathcal{H}^{1}\left(\boldsymbol{A}^{0} \boldsymbol{A}^{1}\right)^{p}$ and $\mathcal{H}^{1}\left(\boldsymbol{A}^{0} \boldsymbol{B}^{1}\right)^{p}$, namely

$$
\begin{aligned}
\mathcal{H}^{1}\left(\boldsymbol{A}^{0} \boldsymbol{A}^{1}\right)^{p} & =\mathcal{H}^{1}\left(A^{0} A^{1}\right)^{p-1} \int_{A^{0} A^{1}}|D \varphi|^{p} d \mathcal{H}^{1}, \\
\mathcal{H}^{1}\left(\boldsymbol{A}^{0} \boldsymbol{B}^{1}\right)^{p} & =\mathcal{H}^{1}\left(A^{0} A^{1}\right)^{p-1} \int_{A^{0} B^{1}}|D \varphi|^{p} d \mathcal{H}^{1} .
\end{aligned}
$$

By inserting both of these equations into (2-4) and using (2-1) with $r=\mathcal{H}^{1}\left(A^{0} A^{1}\right)$, one gets

$$
\left|D^{b} \tilde{h}\right|^{p} \leq \frac{2^{p}}{\mathcal{H}^{1}\left(A^{0} A^{1}\right)} \int_{B\left(A^{0}, \mathcal{H}^{1}\left(A^{0} A^{1}\right)\right) \cap \partial Q}|D \varphi|^{p} d \mathcal{H}^{1} \leq 2^{p} \widetilde{K} \int_{\partial Q}|D \varphi|^{p} d \mathcal{H}^{1} .
$$

Summarizing, in the nondegenerate case, we can finally deduce (2-3) from

$$
\begin{aligned}
\int_{\mathcal{T}}|D \tilde{h}|^{p} & =\frac{\mathcal{H}^{1}\left(A^{0} A^{1}\right)^{2}}{2}|D \tilde{h}|^{p} \\
& \leq \frac{\mathcal{H}^{1}\left(A^{0} A^{1}\right)^{2}}{2} 2^{p} \tilde{K} \int_{\partial Q}|D \varphi|^{p} d \mathcal{H}^{1} \leq 2^{p} \tilde{K} \int_{\partial Q}|D \varphi|^{p} d \mathcal{H}^{1} .
\end{aligned}
$$

Now, let $\tilde{h}$ be a degenerate affine function on each of the two parts $A^{1} P A^{0}$ and $A^{0} P B^{1}$, where $P$ satisfies $\tilde{h}(P)=A^{0}$, as in Step V. Let us call $\left|D^{l} \tilde{h}\right|$ and $\left|D^{r} \tilde{h}\right|$ the constant values of $|D \tilde{h}|$ on the two parts. In this case the following relations hold:

$$
\begin{aligned}
\left|D_{1}^{r} \tilde{h}\right|=\left|D_{1}^{l} \tilde{h}\right|=\frac{\mathcal{H}^{1}\left(\boldsymbol{A}^{0} \boldsymbol{A}^{1}\right)+\mathcal{H}^{1}\left(\boldsymbol{A}^{0} \boldsymbol{B}^{1}\right)}{\mathcal{H}^{1}\left(A^{1} B^{1}\right)} & =\frac{\mathcal{H}^{1}\left(\boldsymbol{A}^{0} \boldsymbol{A}^{1}\right)+\mathcal{H}^{1}\left(\boldsymbol{A}^{0} \boldsymbol{B}^{1}\right)}{\sqrt{2} \mathcal{H}^{1}\left(A^{1} A^{0}\right)}, \\
\frac{\sqrt{2}}{2}\left|D_{2}^{l} \tilde{h}-D_{1}^{l} \tilde{h}\right| & =\frac{\mathcal{H}^{1}\left(\boldsymbol{A}^{0} \boldsymbol{A}^{1}\right)}{\mathcal{H}^{1}\left(A^{0} A^{1}\right)}, \\
\frac{\sqrt{2}}{2}\left|D_{1}^{r} \tilde{h}+D_{2}^{r} \tilde{h}\right| & =\frac{\mathcal{H}^{1}\left(\boldsymbol{A}^{0} \boldsymbol{B}^{1}\right)}{\mathcal{H}^{1}\left(A^{0} B^{1}\right)} .
\end{aligned}
$$

Therefore

$$
\left|D_{2}^{r} \tilde{h}\right| \leq \frac{3 \mathcal{H}^{1}\left(\boldsymbol{A}^{0} \boldsymbol{B}^{1}\right)+\mathcal{H}^{1}\left(\boldsymbol{A}^{0} \boldsymbol{A}^{1}\right)}{\sqrt{2} \mathcal{H}^{1}\left(A^{1} A^{0}\right)},
$$

and in particular

$$
\begin{aligned}
\left|D^{r} \tilde{h}\right|^{p} & \leq \frac{1}{\left(\sqrt{2} \mathcal{H}^{1}\left(A^{1} A^{0}\right)\right)^{p}}\left(4^{p} \mathcal{H}^{1}\left(\boldsymbol{A}^{0} \boldsymbol{A}^{1}\right)^{p}+8^{p} \mathcal{H}^{1}\left(\boldsymbol{A}^{0} \boldsymbol{B}^{1}\right)^{p}\right) \\
& \leq 2 \frac{8^{p}}{\sqrt{2}^{p}} \frac{1}{\mathcal{H}^{1}\left(A^{1} A^{0}\right)} \int_{B\left(A^{0}, \mathcal{H}^{1}\left(A^{1} A^{0}\right)\right) \cap \partial Q}|D \varphi|^{p} d \mathcal{H}^{1} \\
& \leq 2 \frac{8^{p}}{\sqrt{2}^{p}} \widetilde{K} \int_{\partial Q}|D \varphi|^{p} d \mathcal{H}^{1},
\end{aligned}
$$


where the last inequality is a consequence of Step I. An estimate for $\left|D^{l} \tilde{h}\right|^{p}$ analogous to (2-5) holds by a symmetric argument, then, as a consequence, one has

$$
\begin{aligned}
\int_{A^{0} A^{1} B^{1}}|D \tilde{h}|^{p} & =\int_{A^{0} P A^{1}}\left|D^{l} \tilde{h}\right|^{p}+\int_{A^{0} P B^{1}}\left|D^{r} \tilde{h}\right|^{p} \\
& \leq \frac{\mathcal{H}^{1}\left(A^{1} A^{0}\right)^{2}}{2}\left(\left|D^{r} \tilde{h}\right|^{p}+\left|D^{l} \tilde{h}\right|^{p}\right) \leq 2^{\frac{5}{2} p+1} \widetilde{K} \int_{\partial Q}|D \varphi|^{p} d \mathcal{H}^{1} .
\end{aligned}
$$

Therefore (2-3) holds true for the degenerate case and also in general as soon as $K_{1} \geq 2^{\frac{5}{2} p+1} \widetilde{K}$.

Step VII. Estimate for $\int_{\mathcal{Q}^{-}}|D \tilde{h}|^{p}$. In this step, we show that $\tilde{h}$ satisfies (1-1) also in $\mathcal{Q}^{-}$, which is the square $\mathcal{Q}$ without the top and the bottom triangles. Namely, we prove that

$$
\int_{\mathcal{Q}^{-}}|D \tilde{h}|^{p} \leq K_{2} \int_{\partial \mathcal{Q}}|D \varphi|^{p} d \mathcal{H}^{1}
$$

To do so, we need at first a similar estimate on a generic triangle $\mathcal{T}$ of the triangulation of $\mathcal{Q}$ which is inside $\mathcal{Q}^{-}$. To this end, let $i$ be the index so that $\mathcal{T}$ is included in the polygon $\mathcal{D}_{i}:=A_{i} B_{i} \cup A_{i+1} B_{i+1} \cup A_{i} A_{i+1} \cup B_{i} B_{i+1}$. We aim to show

$$
\int_{\mathcal{T}}|D \tilde{h}|^{p} \leq K^{\prime}|\mathcal{T}| \int_{\partial Q}|D \varphi|^{p} d \mathcal{H}^{1}+K^{\prime \prime} \frac{|\mathcal{T}|}{\left|A_{2}^{i+1}-A_{2}^{i}\right|} \int_{A^{i} A^{i+1} \cup B^{i} B^{i+1}}|D \varphi|^{p} d \mathcal{H}^{1} .
$$

Let $\mathcal{T}$ be of the form $P_{j} P_{j+1} R_{j}$ (of course for the other triangles $P_{j+1} R_{j} R_{j+1}$ the very same argument can be applied) and, by symmetry, let us also assume that $\mathcal{D}_{i}$ lays below the $x$-axis. To simplify the notation, we denote $r$ the distance between $A^{0}$ and the horizontal segment $A^{i+1} B^{i+1}$, and $\sigma$ the distance between $A^{i+1} B^{i+1}$ and $A^{i} B^{i}$ (which is equal to $\left|A_{2}^{i+1}-A_{2}^{i}\right|$ and to the height of $\mathcal{D}_{i}$ ). Since $\tilde{h}$ is affine on $\mathcal{T}$, we also denote by $\left|D^{\mathcal{T}} \tilde{h}\right|$ the constant value of $|D \tilde{h}|$ on $\mathcal{T}$. Arguing similarly to Step VI, we would like to estimate both the components $\left|D_{1}^{\mathcal{T}} \tilde{h}\right|$ and $\left|D_{2}^{\mathcal{T}} \tilde{h}\right|$. It follows by construction that

$$
\left|D_{1}^{\mathcal{T}} \tilde{h}\right|^{p}=\frac{\mathcal{H}^{1}\left(\gamma^{i+1}\right)^{p}}{(2 r)^{p}} .
$$

Since $\gamma^{i+1}$ is defined to be the shortest path in $\overline{\mathcal{Q}}$ connecting $\boldsymbol{A}^{i+1}$ to $\boldsymbol{B}^{i+1}$, then in particular it is shorter than the image through $\varphi$ of the curve connecting $A^{i+1}$ to $B^{i+1}$ on $\partial \mathcal{Q}$ passing through $A^{0}$. Therefore, it satisfies the inequality

$$
\mathcal{H}^{1}\left(\gamma^{i+1}\right) \leq \int_{B\left(A^{0}, r \sqrt{2}\right) \cap \partial \mathcal{Q}}|D \varphi| d \mathcal{H}^{1} .
$$

By Hölder's inequality and (2-1) we obtain

$$
\mathcal{H}^{1}\left(\gamma^{i+1}\right)^{p} \leq(2 r \sqrt{2})^{p / p^{\prime}} \int_{B\left(A^{0}, r \sqrt{2}\right) \cap \partial \mathcal{Q}}|D \varphi|^{p} d \mathcal{H}^{1},
$$


and

$$
\left|D_{1}^{\mathcal{T}} \tilde{h}\right|^{p} \leq \frac{\sqrt{2}^{p / p^{\prime}}}{2} \frac{1}{r} \int_{B\left(A^{0}, r \sqrt{2}\right) \cap \partial \mathcal{Q}}|D \varphi|^{p} d \mathcal{H}^{1} \leq \frac{\sqrt{2}^{p / p^{\prime}}}{2} \widetilde{K} \int_{\partial \mathcal{Q}}|D \varphi|^{p} d \mathcal{H}^{1}
$$

where $p^{\prime}$ is such that $\frac{1}{p}+\frac{1}{p^{\prime}}=1$. Estimating $D_{2}^{\mathcal{T}} \tilde{h}$ is less straightforward. Let us call

$$
d:=\mathcal{H}^{1}\left(A^{i+1} P_{j}\right), \quad d^{\prime}:=\mathcal{H}^{1}\left(A^{i} Q_{j}\right), \quad \ell:=\max \left\{\mathcal{H}^{1}\left(\boldsymbol{A}^{i} \boldsymbol{A}^{i+1}\right), \mathcal{H}^{1}\left(\boldsymbol{B}^{i} \boldsymbol{B}^{i+1}\right)\right\}
$$

Then we can write

$$
\left|\left(d^{\prime}+\sigma-d\right) D_{1}^{\mathcal{T}} \tilde{h}+\sigma D_{2}^{\mathcal{T}} \tilde{h}\right|=\mathcal{H}^{1}\left(\boldsymbol{P}_{j} \boldsymbol{Q}_{j}\right) \leq \ell
$$

and some geometrical considerations lead to an estimate of the term $\left|d-d^{\prime}\right|\left|D_{1}^{\mathcal{T}} \tilde{h}\right|$. Indeed, the path $\gamma^{i+1}$ is shorter than $\left.\left.\boldsymbol{A}^{i} \boldsymbol{A}^{i+1} \cup \gamma^{i}\right|_{\boldsymbol{A}^{i} \boldsymbol{Q}_{j}} \cup \boldsymbol{Q}_{j} \boldsymbol{P}_{j} \cup \gamma^{i+1}\right|_{\boldsymbol{P}_{j} \boldsymbol{B}^{i+1}}$, providing that

$$
\mathcal{H}^{1}\left(\gamma^{i+1}\right) \leq 2 \ell+\mathcal{H}^{1}\left(\gamma^{i}\right) \frac{d^{\prime}}{\mathcal{H}^{1}\left(A^{i} B^{i}\right)}+\mathcal{H}^{1}\left(\gamma^{i+1}\right)\left(1-\frac{d}{\mathcal{H}^{1}\left(A^{i+1} B^{i+1}\right)}\right),
$$

which gives in particular

$$
d \frac{\mathcal{H}^{1}\left(\gamma^{i+1}\right)}{\mathcal{H}^{1}\left(A^{i+1} B^{i+1}\right)}-d^{\prime} \frac{\mathcal{H}^{1}\left(\gamma^{i}\right)}{\mathcal{H}^{1}\left(A^{i} B^{i}\right)} \leq 2 \ell .
$$

Since the symmetric argument involving $\gamma^{i}$ gives the opposite inequality (this time we use that $\gamma^{i}$ is shorter than $\left.\left.\boldsymbol{A}^{i} \boldsymbol{A}^{i+1} \cup \gamma^{i+1}\right|_{\boldsymbol{A}^{i+1} \boldsymbol{P}_{j}} \cup \boldsymbol{P}_{j} \boldsymbol{Q}_{j} \cup \gamma^{i}\right|_{\boldsymbol{Q}_{j} \boldsymbol{B}^{i}}$, we get

$$
\left|d \frac{\mathcal{H}^{1}\left(\gamma^{i+1}\right)}{\mathcal{H}^{1}\left(A^{i+1} B^{i+1}\right)}-d^{\prime} \frac{\mathcal{H}^{1}\left(\gamma^{i}\right)}{\mathcal{H}^{1}\left(A^{i} B^{i}\right)}\right| \leq 2 \ell .
$$

Moreover, recalling that $\gamma^{i+1}$ is parametrized at constant speed, it follows directly that

$$
\begin{aligned}
\left|d-d^{\prime}\right|\left|D_{1}^{\mathcal{T}} \tilde{h}\right| & \leq 2 \ell+d^{\prime}\left|\frac{\mathcal{H}^{1}\left(\gamma^{i+1}\right)}{\left.\mathcal{H}^{(} A^{i+1} B^{i+1}\right)}-\frac{\mathcal{H}^{1}\left(\gamma^{i}\right)}{\left.\mathcal{H}^{(} A^{i} B^{i}\right)}\right| \\
& \leq 2 \ell+\left|\frac{\left.\mathcal{H}^{(} A^{i} B^{i}\right)}{\left.\mathcal{H}^{(} A^{i+1} B^{i+1}\right)} \mathcal{H}^{1}\left(\gamma^{i+1}\right)-\mathcal{H}^{1}\left(\gamma^{i}\right)\right| \\
& \leq 2 \ell+\left|\mathcal{H}^{1}\left(\gamma^{i+1}\right)-\mathcal{H}^{1}\left(\gamma^{i}\right)\right|+2 \sigma \frac{\mathcal{H}^{1}\left(\gamma^{i+1}\right)}{\left.\mathcal{H}^{(} A^{i+1} B^{i+1}\right)} \leq 4 \ell+2 \sigma\left|D_{1}^{\mathcal{T}} \tilde{h}\right| .
\end{aligned}
$$

Inserting, then, the above estimate into (2-8), we get

$$
\left|D_{2}^{\mathcal{T}} \tilde{h}\right| \leq \frac{5}{\sigma} \ell+3\left|D_{1}^{\mathcal{T}} \tilde{h}\right|,
$$


which in turn implies

$$
\left|D_{2}^{\mathcal{T}} \tilde{h}\right|^{p} \leq\left(\frac{10 l}{\sigma}\right)^{p}+6^{p}\left|D_{1}^{\mathcal{T}} \tilde{h}\right|^{p}
$$

Let us notice that we can easily bound $\ell^{p}$ from above since $\varphi$ is linear on $A^{i} A^{i+1}$ and $B^{i} B^{i+1}$. Indeed, let $\ell$ be for instance equal to $\mathcal{H}^{1}\left(A^{i} A^{i+1}\right)$; then

$$
\ell^{p}=(\sigma \sqrt{2})^{p-1} \int_{A^{i} A^{i+1}}|D \varphi|^{p} d \mathcal{H}^{1} \leq(\sigma \sqrt{2})^{p-1} \int_{A^{i} A^{i+1} \cup B^{i} B^{i+1}}|D \varphi|^{p} d \mathcal{H}^{1}
$$

where we used that $\mathcal{H}^{1}\left(A^{i} A^{i+1}\right)=\sigma \sqrt{2}$. Thus, by inserting (2-10) and (2-7) into (2-9) one gets

$$
\left|D_{2}^{\mathcal{T}} \tilde{h}\right|^{p} \leq 6^{p} \frac{\sqrt{2}^{p / p^{\prime}}}{2} \widetilde{K} \int_{\partial Q}|D \varphi|^{p} d \mathcal{H}^{1}+\frac{(10 \sqrt{2})^{p}}{2} \frac{1}{\sigma} \int_{A^{i} A^{i+1} \cup B^{i} B^{i+1}}|D \varphi|^{p} d \mathcal{H}^{1},
$$

which, together with (2-7), gives (2-6) with $K^{\prime}=6^{p} \frac{1}{2} \sqrt{2}^{p / p^{\prime}} \widetilde{K}$ and $K^{\prime \prime}=\frac{1}{2}(10 \sqrt{2})^{p}$. Moreover, by summing up among all the triangles $\mathcal{T}$ in $\mathcal{D}_{i}$ and observing that $\left|\mathcal{D}_{i}\right| \leq 2 \sigma$ by construction, we have

$$
\begin{aligned}
\int_{\mathcal{D}_{i}}|D \tilde{h}|^{p} & \leq K^{\prime}\left|\mathcal{D}_{i}\right| \int_{\partial \mathcal{Q}}|D \varphi|^{p} d \mathcal{H}^{1}+K^{\prime \prime} \frac{\left|\mathcal{D}_{i}\right|}{\sigma} \int_{A^{i} A^{i+1} \cup B^{i} B^{i+1}}|D \varphi|^{p} d \mathcal{H}^{1} \\
& \leq K^{\prime}\left|\mathcal{D}_{i}\right| \int_{\partial \mathcal{Q}}|D \varphi|^{p} d \mathcal{H}^{1}+2 K^{\prime \prime} \int_{A^{i} A^{i+1} \cup B^{i} B^{i+1}}|D \varphi|^{p} d \mathcal{H}^{1} .
\end{aligned}
$$

Finally, on the whole $\mathcal{Q}^{-}$one gets

$$
\begin{aligned}
\int_{\mathcal{Q}^{-}}|D \tilde{h}|^{p} & \leq K^{\prime}\left|\mathcal{Q}^{-}\right| \int_{\partial \mathcal{Q}}|D \varphi|^{p} d \mathcal{H}^{1}+2 K^{\prime \prime} \int_{\partial \mathcal{Q}^{-} \cap \partial \mathcal{Q}}|D \varphi|^{p} d \mathcal{H}^{1} \\
& \leq K_{2} \int_{\partial \mathcal{Q}}|D \varphi|^{p} d \mathcal{H}^{1},
\end{aligned}
$$

for a suitable $K_{2} \geq 2 \max \left\{K^{\prime}, K^{\prime \prime}\right\}$.

Step VIII. Definition of $h$ and conclusion. We can now observe that, whenever $\tilde{h}$ is a homeomorphism, Theorem 1.1 follows directly. Indeed, $\tilde{h}$ coincides with $\varphi$ on $\partial \mathcal{Q}$, it is finitely piecewise affine and, moreover, the estimates (2-3) and (2-11) provide that (1-1) is satisfied by $\tilde{h}$ and $K \geq \max \left\{K_{1}, K_{2}\right\}$. Unfortunately, in our construction the function $\tilde{h}$ happens to be one-to-one only when all the paths $\gamma^{i}$ lie in the interior of $\mathcal{Q}$ without intersecting each other. Of course in general this is not the case, but it is always possible to slightly modify the paths $\gamma^{i}$ in order to get the one-to-one property. A possible configuration can be seen Figure 4. More precisely, it is always possible to separate a curve $\gamma^{i+1}$ from either $\partial \mathcal{Q}$ and $\gamma^{i}$ so that the minimal distance between them is much smaller than the lengths of all the linear pieces of the paths $\gamma$ and $\partial \mathcal{Q}$. Let us notice that the minimal distance is 

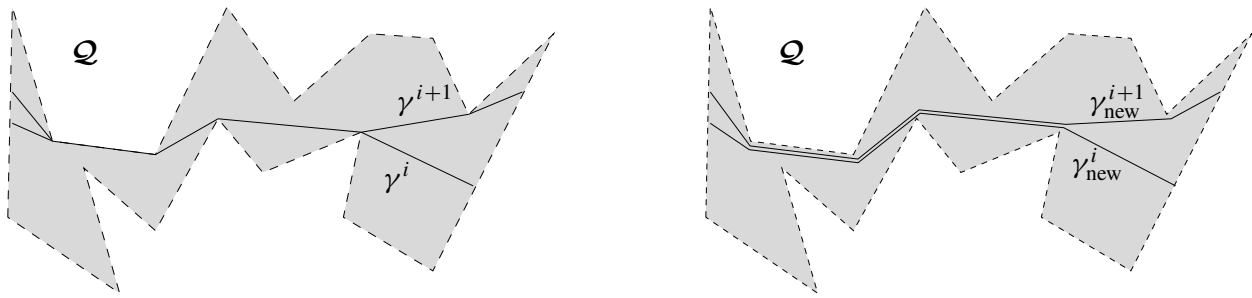

Figure 4. Modification of $\gamma^{i}$ and $\gamma^{i+1}$.

strictly positive because there is only a finite number of paths and each of them is finitely piecewise linear. We finally define the function $h$ in the very same way as we defined $\tilde{h}$ in Step V, but this time using the separated paths. Therefore, the function $h$ is a homeomorphism, it is still finitely piecewise affine, it satisfies the boundary condition on $\partial \mathcal{Q}$ and, furthermore, estimate (1-1) is still valid up to increasing the geometric constant $K$ by a quantity which is as small as we wish. This implies the validity of Theorem 1.1 and concludes the proof.

Remark 2.1. Let $\mathcal{Q} \subset \mathbb{R}^{2}$ be a generic square of length side $r, p \geq 1$ and $\varphi: \partial \mathcal{Q} \rightarrow \mathbb{R}^{2}$ piecewise linear. Then there exists a piecewise affine function $h: \mathcal{Q} \rightarrow \mathbb{R}^{2}$ that coincides with $\varphi$ on the boundary $\partial \mathcal{Q}$ and a geometric constant $K$ depending only on $p$ such that

$$
\int_{\mathcal{Q}}|D h|^{p} \leq K r \int_{\partial \mathcal{Q}}|D \varphi|^{p} d \mathcal{H}^{1}
$$

Indeed, there always exists an affine function $\rho$ mapping the unit square $\mathcal{Q}_{1}$ onto $\mathcal{Q}$. Let us call, with a slight abuse of notation, $\rho$ and its restriction to the boundary $\partial \mathcal{Q}_{1}$ with the same name. Then, by applying Theorem 1.1 to the function $\varphi \circ \rho$ and recalling that $|D \rho|=r$, it is possible to find a constant $K$ and a piecewise affine function $\tilde{h}: \mathcal{Q}_{1} \rightarrow \mathbb{R}^{2}$ satisfying

$$
\int_{\mathcal{Q}_{1}}|D \tilde{h}(x)|^{p} d x \leq K \int_{\partial \mathcal{Q}_{1}} r^{p}|D \varphi(\rho(t))|^{p} d \mathcal{H}^{1}(t) .
$$

Finally, by defining $h:=\tilde{h} \circ \rho^{-1}$ and changing the variables, one gets

$$
r^{p-2} \int_{\mathcal{Q}}|D h|^{p} \leq r^{p-1} K \int_{\partial \mathcal{Q}}|D \varphi|^{p} d \mathcal{H}^{1}
$$

and (2-12) follows.

\section{References}

[Bellido and Mora-Corral 2011] J. C. Bellido and C. Mora-Corral, "Approximation of Hölder continuous homeomorphisms by piecewise affine homeomorphisms", Houston J. Math. 37:2 (2011), 449-500. MR 2794559 Zbl 1228.57009 
[Campbell 2015] D. Campbell, "Diffeomorphic approximation of planar Sobolev homeomorphisms in Orlicz-Sobolev spaces", preprint, 2015, available at http://tinyurl.com/grptrrc.

[Daneri and Pratelli 2015] S. Daneri and A. Pratelli, "A planar bi-Lipschitz extension theorem", Adv. Calc. Var. 8:3 (2015), 221-266. MR 3365742 Zbl 1331.26020

[Hencl and Pratelli 2015] S. Hencl and A. Pratelli, "Diffeomorphic approximation of $W^{1,1}$ planar Sobolev homeomorphisms", preprint, 2015, available at http://arxiv.org/abs/1502.07253.

[Iwaniec et al. 2011] T. Iwaniec, L. V. Kovalev, and J. Onninen, "Diffeomorphic approximation of Sobolev homeomorphisms”, Arch. Ration. Mech. Anal. 201:3 (2011), 1047-1067. MR 2824471 Zbl 1260.46023

[Mora-Corral 2009] C. Mora-Corral, "Approximation by piecewise affine homeomorphisms of Sobolev homeomorphisms that are smooth outside a point", Houston J. Math. 35:2 (2009), 515-539. MR 2519545 Zbl 1182.57019

Received August 26, 2015. Revised November 25, 2015.

EMANUELA RADICI

DEPARTMENT OF MATHEMATICS

UNIVERSITY OF ERLANGEN

CAuerstrasse 11

D-90158 ERLANGEN

GERMANY

radici@math.fau.de 


\title{
PACIFIC JOURNAL OF MATHEMATICS
}

Founded in 1951 by E. F. Beckenbach (1906-1982) and F. Wolf (1904-1989)

$$
\text { msp.org/pjm }
$$

\section{EDITORS}

\author{
Don Blasius (Managing Editor) \\ Department of Mathematics \\ University of California \\ Los Angeles, CA 90095-1555 \\ blasius@math.ucla.edu
}

\author{
Paul Balmer \\ Department of Mathematics \\ University of California \\ Los Angeles, CA 90095-1555 \\ balmer@math.ucla.edu \\ Robert Finn \\ Department of Mathematics \\ Stanford University \\ Stanford, CA 94305-2125 \\ finn@math.stanford.edu \\ Sorin Popa \\ Department of Mathematics \\ University of California \\ Los Angeles, CA 90095-1555 \\ popa@math.ucla.edu
}

\author{
Vyjayanthi Chari \\ Department of Mathematics \\ University of California \\ Riverside, CA 92521-0135 \\ chari@math.ucr.edu \\ Kefeng Liu \\ Department of Mathematics \\ University of California \\ Los Angeles, CA 90095-1555 \\ liu@math.ucla.edu \\ Igor Pak \\ Department of Mathematics \\ University of California \\ Los Angeles, CA 90095-1555 \\ pak.pjm@gmail.com \\ Paul Yang \\ Department of Mathematics \\ Princeton University \\ Princeton NJ 08544-1000 \\ yang@math.princeton.edu
}

\section{PRODUCTION}

Silvio Levy, Scientific Editor, production@msp.org

\section{SUPPORTING INSTITUTIONS}

ACADEMIA SINICA, TAIPEI

CALIFORNIA INST. OF TECHNOLOGY

STANFORD UNIVERSITY

UNIV. OF BRITISH COLUMBIA

UNIV. OF CALIFORNIA, BERKELEY

UNIV. OF CALIFORNIA, DAVIS

UNIV. OF CALIFORNIA, LOS ANGELES

UNIV. OF CALIFORNIA, RIVERSIDE

UNIV. OF CALIFORNIA, SAN DIEGO

UNIV. OF CALIF., SANTA BARBARA
KEIO UNIVERSITY

MATH. SCIENCES RESEARCH INSTITUTE

NEW MEXICO STATE UNIV.

OREGON STATE UNIV.
Daryl Cooper

Department of Mathematics

University of California

Santa Barbara, CA 93106-3080 cooper@math.ucsb.edu

Jiang-Hua Lu

Department of Mathematics

The University of Hong Kong

Pokfulam Rd., Hong Kong

jhlu@maths.hku.hk

$$
\text { Jie Qing }
$$

Department of Mathematics

University of California

Santa Cruz, CA 95064

qing@ cats.ucsc.edu

\author{
UNIV. OF CALIF., SANTA CRUZ \\ UNIV. OF MONTANA \\ UNIV. OF OREGON \\ UNIV. OF SOUTHERN CALIFORNIA \\ UNIV. OF UTAH \\ UNIV. OF WASHINGTON \\ WASHINGTON STATE UNIVERSITY
}

These supporting institutions contribute to the cost of publication of this Journal, but they are not owners or publishers and have no responsibility for its contents or policies.

See inside back cover or msp.org/pjm for submission instructions.

The subscription price for 2016 is US $\$ 440 /$ year for the electronic version, and \$600/year for print and electronic.

Subscriptions, requests for back issues and changes of subscriber address should be sent to Pacific Journal of Mathematics, P.O. Box 4163, Berkeley, CA 94704-0163, U.S.A. The Pacific Journal of Mathematics is indexed by Mathematical Reviews, Zentralblatt MATH, PASCAL CNRS Index, Referativnyi Zhurnal, Current Mathematical Publications and Web of Knowledge (Science Citation Index).

The Pacific Journal of Mathematics (ISSN 0030-8730) at the University of California, c/o Department of Mathematics, 798 Evans Hall \#3840, Berkeley, CA 94720-3840, is published twelve times a year. Periodical rate postage paid at Berkeley, CA 94704, and additional mailing offices. POSTMASTER: send address changes to Pacific Journal of Mathematics, P.O. Box 4163, Berkeley, CA 94704-0163.

PJM peer review and production are managed by EditFLOW ${ }^{\circledR}$ from Mathematical Sciences Publishers.

PUBLISHED BY

\section{I. mathematical sciences publishers}

nonprofit scientific publishing

http://msp.org/

(C) 2016 Mathematical Sciences Publishers 


\section{PACIFIC JOURNAL OF MATHEMATICS}

Volume $283 \quad$ No. $2 \quad$ August 2016

The fundamental theorem of tropical differential algebraic geometry

FUENSANTA AROCA, CRISTHIAN GARAY and ZEINAB TOGHANI

A simple solution to the word problem for virtual braid groups

271

PaOlo Bellingeri, Bruno A. Cisneros de la CruZ and Luis

PARIS

Completely contractive projections on operator algebras

DAVID P. BLECHER and MATTHEW NEAL

Invariants of some compactified Picard modular surfaces and applications

AMIR DŽAMBIĆ

Radial limits of bounded nonparametric prescribed mean curvature surfaces

MOZHGAN (NORA) ENTEKHABI and KIRK E. LANCASTER

A remark on the Noetherian property of power series rings

BYUNG GYUN KANG and PHAN THANH TOAN

Curves with prescribed intersection with boundary divisors in moduli spaces of curves

\section{XIAO-LEI LIU}

Virtual rational Betti numbers of nilpotent-by-abelian groups

BEHROOZ MiRZAII and FATEMEH Y. MOKARI

A planar Sobolev extension theorem for piecewise linear homeomorphisms 405

EMANUELA RADICI

A combinatorial approach to Voiculescu's bi-free partial transforms

PAUL SKOUFRANIS

Vector bundle valued differential forms on $\mathbb{N} Q$-manifolds

LUCA VitAGLiANO

Discriminants and the monoid of quadratic rings 